OPEN ACCESS

Edited by:

Antonino F. Germano',

University of Messina, Italy

Reviewed by:

Giuseppe Maria Della Pepa,

Fondazione Policlinico Universitario Agostino Gemelli IRCSS, Italy

domenico D'Avella,

University of Padua, Italy

*Correspondence:

Francesco Sala

francesco.sala@univr.it

Specialty section:

This article was submitted to Neuro-Oncology and Neurosurgical

Oncology,

a section of the journal

Frontiers in Neurology

Received: 28 March 2021

Accepted: 26 May 2021

Published: 28 June 2021

Citation:

Sala F, Giampiccolo D and Cattaneo L (2021) Novel Asleep Techniques for Intraoperative Assessment of Brain

Front. Neurol. 12:687030 doi: 10.3389/fneur.2021.687030

\section{Novel Asleep Techniques for Intraoperative Assessment of Brain Connectivity}

\author{
Francesco Sala $^{1 *}$, Davide Giampiccolo ${ }^{1}$ and Luigi Cattaneo ${ }^{2}$ \\ ${ }^{1}$ Section of Neurosurgery, Department of Neuroscience, Biomedicine and Movement Sciences, University of Verona, Verona, \\ Italy, ${ }^{2} \mathrm{CIMeC}$ - Center for Mind/Brain Sciences, University of Trento, Trento, Italy
}

Keywords: intraoperative neurophysiological monitoring, motor evoked potentials, cortico-cortical evoked potentials, neuro-oncology - surgical, brain mapping

\section{INTRODUCTION}

\section{State of the Art in Intraoperative Monitoring for Neurosurgery and Current Limitations}

From the pioneering work of neurosurgeon-neuroscientists such as Otfried Foerster and Wilder Penfield, mapping of brain function using electrical stimulation has allowed to identify and spare motor behavior and language during awake surgery, producing the first cartographies of the brain cortex $(1,2)$. This has been refined in the early 2000 s as neurosurgeons started routine subcortical awake mapping using white matter tracts as subcortical boundaries (3), with subsequent improvement in both functional (4) and surgical outcome $(5,6)$. The advantages of performing awake surgery when cognitive functions are at risk, should not be questioned, and we remark this should be performed whenever feasible. However, patients with pre-existing neurological deficits and/or inadequate neuropsychological profiles are not good candidates for awake surgery, and therefore must undergo asleep procedures. Notably, pediatric patients cannot undergo awake surgery, on one hand because of their scarce compliance (7), on the other hand because the immaturity of their motor system makes the cortex almost inexcitable using the traditional bipolar Penfield's 50/60 Hz technique $(8,9)$.

In patients who are poor candidates for awake surgery, developing methods to map cortico-cortical and cortico-subcortical connectivity under anesthesia is of primary importance since otherwise surgery will be performed blind to function, with higher risk of incurring into neurological deficits. Since the adoption of somatosensory evoked potentials (SEPs) in the "70s (10) and particularly with the development of the train-of-five motor evoked potential (MEP) technique (11), the field of intraoperative neurophysiological monitoring (IONM) has specifically addressed the issue of mapping and monitoring during asleep anesthesia. Standard protocols for motor mapping are nowadays available, offering reproducible, and reliable parameters to qualitatively and quantitatively predict outcome (12). This somehow differed from awake surgery, where neuropsychological tests and mapping protocols have a greater degree of variability. In brain tumor surgery IONM is particularly well-established for preservation of the corticospinal tract $(13,14)$, but asleep mapping and monitoring outside the corticospinal system is lacking. The aim of this opinion paper is to discuss two novel potential IONM techniques allowing to map and monitor functions beyond corticospinal motor function in the anesthetized setting, namely, (A) conditioning of motor output $(15,16)$ and $(B)$ cortico-cortical evoked potentials $(17,18)$. These have been performed under total intravenous anesthesia using Propofol (100-150 $\mu \mathrm{g} / \mathrm{kg} / \mathrm{min})$ and Fentanyl $(1 \mu \mathrm{g} / \mathrm{kg} / \mathrm{min})$ in continuous infusion, and avoiding muscle relaxants after intubation. 


\section{Potential Novel Intraoperative Measures of Brain Connectivity in the Anesthetized Patient \\ Conditioning of Corticospinal Output}

The conditioning stimulus (CS)/test stimulus (TS) paradigms have been used widely in experimental and clinical neurophysiology to investigate functional connectivity between two regions of the nervous system. In CS/TS paradigms (illustrated in panel A of Figure 1), a suprathreshold stimulus (TS) is delivered to the motor cortex, thus evoking a motor potential (MEP) of a given amplitude. The CS, of intensity comparable to that of the TS, is delivered to a region that is supposedly connected to the motor cortex but is not itself a source of corticospinal output. Therefore, the CS alone does not produce a MEP. However, when the CS precedes the TS, the MEP obtained by the conditioned TS may be different (i.e., increased or decreased MEP amplitude) from that obtained by TS alone. Whenever such remote effects of CS on TS occur, they are taken as evidence of functional connectivity between the site of application of the CS and that of the TS. The CS-TS interactions are generally specific for given inter-stimulus intervals (ISI), i.e., the time interval between CS and TS. Conditioning effects of the CS over the TS at short ISIs is generally thought to be informative of the underlying anatomical connections: interactions at short ISIs indicate direct cortico-cortical connections.

CS/TS paradigms are commonly explored using non-invasive brain stimulation, namely transcranial magnetic stimulation (TMS) applied with two different coils over the scalp, one delivering the CS and the other delivering the TS over the motor cortex (20). These paradigms have been extensively reviewed by Koch (21). The modulatory effect of CS can be excitatory or inhibitory, therefore increasing or decreasing MEP amplitude compared to TS alone. However, as MEP amplitude can physiologically vary between two identical stimuli, it is important to repeat both TS stimulations and CS+TS stimulation. Therefore, the comparison between CS+TS MEPs and MEPs to TS alone cannot be done between single MEPs, but must be performed between groups of conditioned (CS+TS) MEPs and of baseline (TS alone) MEPs.

The descending corticospinal volley evoked by stimulation of the motor cortex has different components, separated in time. The earliest volley is referred to as direct, or " $\mathrm{D}$ " wave and is due to direct activation of corticospinal axons. The later components, known as indirect or "I" waves originate from stimulation of neurons that in turn project onto the corticospinal neurons, which are therefore activated trans-synaptically (22).

\footnotetext{
Abbreviations: AF, arcuate fasciculus; CCEPs, Cortico-cortical evoked potentials; CS, conditioning stimulus; CST, cortico-spinal tract; DES, direct electrical stimulation; DTI, diffusion tensor imaging; DWI, diffusion weighted imaging; ECoG, electrocorticography; EEG, electroencephalography; EMG, electromyography; HARDI, high angular resolution diffusion imaging; ISI, interstimulus interval; IONM, intraoperative neurophysiological monitoring; MEPs, motor evoked potentials; MFG, middle frontal gyrus; mFUSA, middle fusiform gyrus; MTG, middle temporal gyrus; pOP, pars opercularis; pTRI, pars triangularis; STG, superior temporal gyrus; TOj, temporo-occipital junction; To5, train-of-five stimulation; TS, test stimulus; vPM, ventral premotor cortex; ITG, inferior temporal gyrus.
}

It is important to note that for the TS to be susceptible to modulation by the CS, it must produce a corticospinal volley containing I-waves, as the $\mathrm{D}$-wave cannot be modulated by any afferents to the corticospinal neuron because it is generated downstream of any point of integration of inputs from cortico-cortical afferents. Indeed, direct cortical stimulation and intraoperative transcranial electrical stimulation are known to produce I-waves (23-25).

We propose that the CS/TS approach can be successfully performed also by means of direct cortical stimulation in the intraoperative setting, and therefore potentially be used for assessing and potentially monitoring the integrity of specific brain and spine connections, as demonstrated by two recent works from our group, on parietal-motor connectivity (15) and on cerebello-motor connectivity (16).

In the first work we described the successful use of a CS/TS paradigm in the intraoperative setting to explore putative direct parietal-motor connectivity (15) (Figure 1B). Briefly, two strip electrodes were deployed, one over the parietal cortex (CS) and one over the motor cortex (TS). Conditioning stimuli in the parietal cortex were always delivered in a short train of 2 stimuli at $250 \mathrm{~Hz}$ and of $0.5 \mathrm{~ms}$ duration while test stimulus varied from a single stimulus to a train of 3 stimuli at $250 \mathrm{~Hz}$ and $0.5 \mathrm{~ms}$ duration according to the individual patient's cortical excitability. Stimulation intensity for conditioning and test stimulation was always the same $(15-35 \mathrm{~mA})$. Prior to all experimental stimulations we acquired blocks of conditioning stimuli alone, verifying that no MEP could be observed from test stimulation

Such functional connectivity has been abundantly described by means of dual coil TMS in healthy participants $(20,26)$. In our study we highlighted the presence of conditioning effects from CS applied directly to the posterior parietal cortex on the TS applied directly to the ipsilateral upper limb motor cortex, in 17 anesthetized oncological patients during surgical resection. The conditioning effects on the TS were specific for both timing and anatomical localization of the CS. The effects appeared at short ISIs $(4-20 \mathrm{~ms})$ with the earliest effective ISI depending on the anatomical proximity of the parietal stimulation electrode to the motor cortex: short ISIs were efficient when the CS was delivered near to the motor cortex. The use of trains of stimuli to stimulate the motor cortex renders timing of the CS/TS ISI difficult. In general we considered, for the sake of timing, the last stimulus of the train as the one that is effective in triggering the efferent corticofugal volley (27). Anatomical specificity was clearly evident in the spatial clustering of CS sites with excitatory effects in the inferior parietal lobule and of CS sites with inhibitory effects in the superior parietal lobule. Note that focality of CS stimulation was granted by the use of bipolar stimulation between adjacent electrodes of a stimulating strip.

In the second work we tested the effects of CS applied to the cerebellar cortex onto corticospinal excitability (16), tested by TS applied transcranially to target the upper limb motor cortex (Figure 1C) in 10 anesthetized patients undergoing posterior fossa tumor surgery. Our experimental paradigm is inspired by a well-established CS+TS technique referred to as 


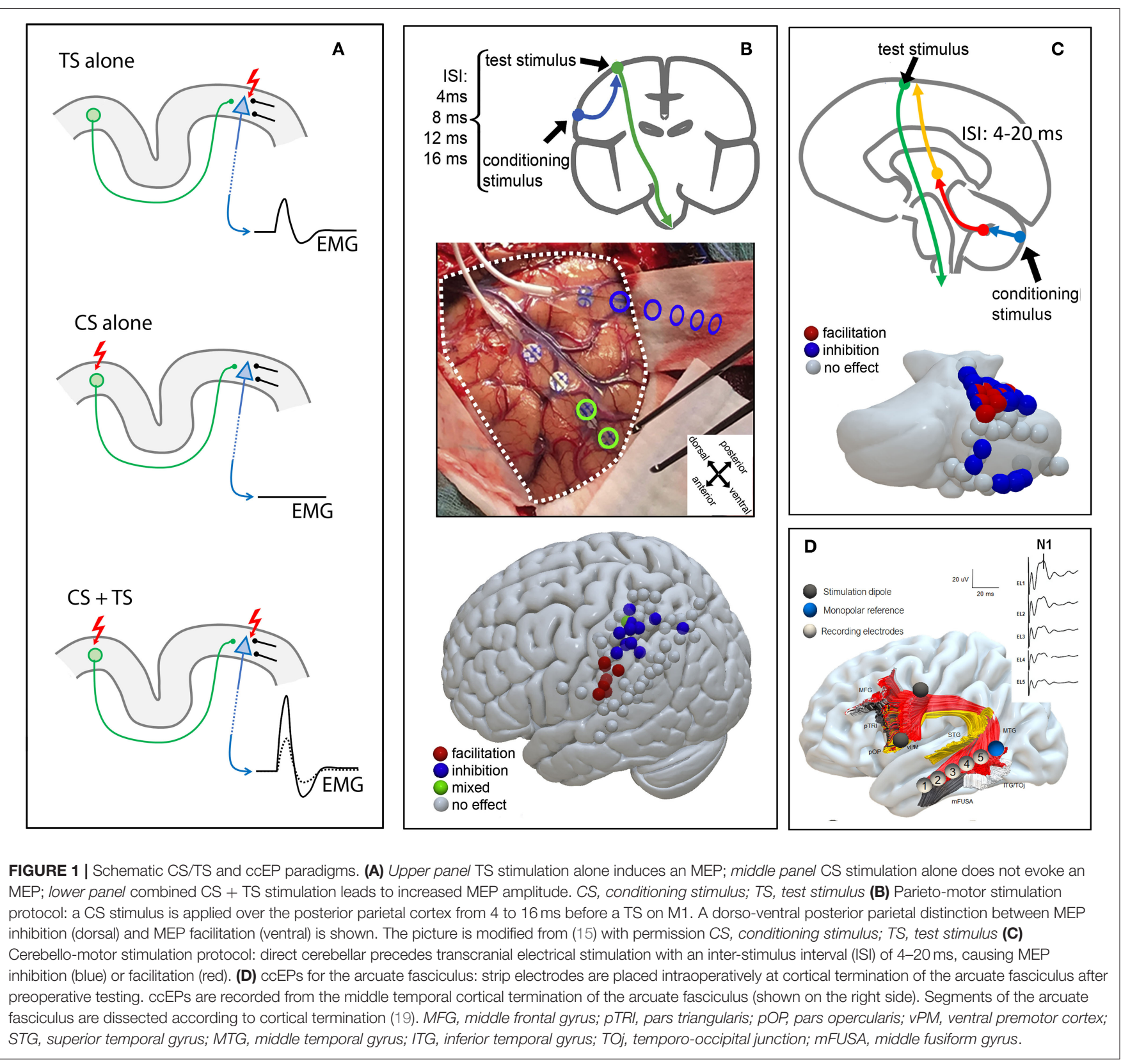

"cerebellar inhibition" in which the cerebellum and the motor cortex are stimulated by TMS $(28,29)$. Briefly, conditioning stimuli on the cerebellar cortex were delivered in a short train of 2-5 stimuli at $250 \mathrm{~Hz}$ and of $0.5 \mathrm{~ms}$ duration with an intensity of $15-25 \mathrm{~ms}$. Test stimuli varied from a train of $2-5$ stimuli at $250 \mathrm{~Hz}$ and $0.5 \mathrm{~ms}$ duration with stimulation intensity of $15-35 \mathrm{~mA}$. Our results showed that CS cerebellar stimuli conditioned at short ISIs the corticospinal excitability, with a significant anatomical specificity: cerebellar CS exerted conditioning effects on the hand corticospinal system when applied to regions of the cerebellar cortex in the anterior and posterior lobule that are known to contain hand representations $(30,31)$.

\section{Cortico-Cortical Evoked Potentials in the Anaesthetized Patient}

While the rationale for describing brain connectivity by means of ccEPs was first discussed by Lord Adrian (32), the clinical use of cortico-cortical evoked potentials was first pioneered by Matsumoto (33). In ccEPs, one of two cortical terminations of a white matter tract is stimulated electrically, and cortical evoked activity is recorded at the other termination in the form of evoked potentials. Twenty to 120 raw traces are conventionally averaged, similarly to cortically recorded somatosensory evoked potentials. ccEPs show two components, an N1 between 20 and $30 \mathrm{~ms}(33,34)$ and a second, later component peaking at $100-$ $150 \mathrm{~ms}$ (33), though some authors claim this later component 
could represent epileptogenic activity instead (35). CcEPs latency should reflect fiber myelination and length $(33,36)$. Moreover, there is strong evidence for directionality in the evoked potentials $(37,38)$. The recording of ccEPs is potentially applicable to the whole cerebral cortex and transcends language function $(39,40)$. However, clinical use of this technique has been historically mainly related to language function.

The recording of ccEPs is generally limited to awake patients, because of (a) the suppression of neural activity due to anesthesia (41), and (b) the chance to identify location for strip electrodes placement using functional mapping $(33,42)$. However, their use is of potential interest in monitoring white matter integrity also in the asleep patient. In a recent work in a cohort of 9 patients with tumors in the left perisylvian area who could not undergo awake surgery, we recorded ccEPs of the arcuate fascicle in anesthetized patients undergoing tumor surgery (18). Results indicated that (a) reliable potentials of small amplitude can be obtained from the arcuate fasciculus also under anesthesia and that (b) strip electrode placement can be made more effective by combining tractographic MR information and presurgical neuronavigated TMS (nTMS). Results in the asleep setting resembled those in the awake setting: an $\mathrm{N} 1$ potential with a latency of $21 \mathrm{~ms}$ was shown, together with an earlier positive potential peaking at $12 \mathrm{~ms}$. In our series, evoked potentials clustered in the middle temporal gyrus while stimulation mainly covered the ventral premotor cortex. Although the arcuate fasciculus has cortical terminations over superior, middle and inferior temporal gyri $(43,44)$, such selectivity may be justified by a layered distribution of its components, particularly in a ventral/dorsal fashion (19, 44). Indeed, ccEP responses for this tract may support this, since location for optimal recorded responses varies according to the stimulated gyrus in the frontal lobe (45). Moreover, the inferior temporal gyrus components of the arcuate fasciculus have not been extensively investigated in this study, which is another limitation to be taken into account.

\section{CONCLUSION AND LIMITATIONS}

Asleep surgery without mapping is blind to function and therefore at highest risk of inducing neurological deficits. We

\section{REFERENCES}

1. Vogt C, and Vogt O. Die vergleichend-architektonische und die vergleichendreizphysiologische Felderung der Großhirnrinde unter besonderer Berücksichtigung der menschlichen. Naturwissenschaften (1926) 14:1190-4.

2. Foerster O, Penfield W. The structural basis of traumatic epilepsy and results of radical operation. Brain. (1930) 53:99-119. doi: 10.1093/brain/53.2.99n

3. Duffau H, Capelle L, Denvil D, Sichez N, Gatignol P, Lopes M, et al. Functional recovery after surgical resection of low grade gliomas in eloquent brain: hypothesis of brain compensation. J Neurol Neurosurg Psychiatry. (2003) 74:901-7. doi: 10.1136/jnnp.74.7.901n

4. De Witt Hamer PC, Robles SG, Zwinderman AH, Duffau H, Berger MS. Impact of intraoperative stimulation brain mapping on glioma surgery outcome: a meta-analysis. J Clin Oncol. (2012) 30:2559-65. doi: 10.1200/JCO.2011.38.4818n

5. Darlix A, Rigau V, Fraisse J, Gozé C, Fabbro M, Duffau H. Postoperative follow-up for selected diffuse low-grade gliomas with WHO grade III/IV foci. Neurology. (2020) 94:e830-41. doi: 10.1212/WNL.000000000000 $8877 n$ believe it is an ethical responsibility to raise awareness of this issue. Therefore, the work presented here lies on this foundation and the attempt to predict, and therefore prevent, neurological deficits in patients who are not good candidates to an awake craniotomy.

However, to be useful in clinical practice these techniques require to (a) be standardized; (b) be deterministic, i.e., allow predictions in individual patients; (c) have a strong predictive value of a given clinical/behavioral aspect. The phenomena that we have described do not satisfy any of the above criteria, therefore further research is needed before a clinical use, if any, can be proposed. Points (a) and (b) require extensive tests for standardization and reproducibility. Regarding point (c), we believe that cortico-motor CS/TS paradigms should be tested also from a constellation of other areas that project the motor cortex, namely the premotor, supplementary motor and somatosensory cortices and their perioperative changes need to be correlated with behavior such as skilled movement and sensorimotor behavior in general. The cerebello-motor CS/TS paradigm's predictive value should be tested specifically on the pediatric population undergoing posterior fossa surgery. This is of overriding importance, as individual age-associated myelination and axonal length (9) may imply significant changes in the optimal parameters (ISI, stimulation intensity) for cerebellocortical modulation. Similarly, ccEPs for language connectivity should be tested for their predictive value in perioperative language disorders.

\section{AUTHOR CONTRIBUTIONS}

FS, DG, and LC: conceptualization and writing - original draft. FS: funding acquisition. FS and LC: supervision. All authors contributed to the article and approved the submitted version.

\section{FUNDING}

This work was supported by the grant NEUROCONNECT issued to FS by the Fondazione Cassa di Risparmio di Verona.

6. Rossi M, Gay L, Ambrogi F, Nibali MC, Sciortino T, Puglisi G, et al. Association of supratotal resection with progression-free survival, malignant transformation, and overall survival in lower-grade gliomas. Neuro Oncol. (2020) 23:812-26. doi: 10.1093/neuonc/noaa225n

7. Gallentine WB, Mikati MA. Intraoperative electrocorticography and cortical stimulation in children. J Clin Neurophysiol. (2009) 26:95-108. doi: 10.1097/WNP.0b013e3181a0339dn

8. Ojemann SG, Berger MS, Lettich E, Ojemann GA. Localization of language function in children: results of electrical stimulation mapping. J Neurosurg. (2003) 98:465-70. doi: 10.3171/jns.2003.98.3.0465n

9. Sala F, Manganotti P, Grossauer S, Tramontanto V, Mazza C, Gerosa M. Intraoperative neurophysiology of the motor system in children: a tailored approach. Child's Nerv Syst. (2010) 26:473-90. doi: 10.1007/s00381-009-1081-6n

10. Tamaki T, Yamashita T, Kobayashi H. Spinal cord monitoring. Jpn J Electroenceph Electromyogr. (1972) 1:196n

11. Taniguchi M, Cedzich C, Schramm J. Modification of cortical stimulation for motor evoked potentials under general anesthesia: technical description. Neurosurgery. (1993) 32:219-26. doi: 10.1227/00006123-199302000-00011n 
12. Seidel K, Beck J, Stieglitz L, Schucht P, Raabe A. The warning-sign hierarchy between quantitative subcortical motor mapping and continuous motor evoked potential monitoring during resection of supratentorial brain tumors. J Neurosurg. (2013) 118:287-96. doi: 10.3171/2012.10.JNS12895n

13. Neuloh G, Pechstein U, Schramm J. Motor tract monitoring during insular glioma surgery. J Neurosurg. (2007) 106:58292. doi: $10.3171 /$ jns.2007.106.4.582n

14. Giampiccolo D, Parisi C, Meneghelli P, Tramontano V, Basaldella F, Pasetto $\mathrm{M}$, et al. Long-term motor deficit in brain tumor surgery with preserved intraoperative motor evoked potentials. Brain Commun. (2021) 3:fcaa226. doi: 10.1093/braincomms/fcaa226n

15. Cattaneo L, Giampiccolo D, Meneghelli P, Tramontano V, Sala F. Corticocortical connectivity between the superior and inferior parietal lobules and the motor cortex assessed by intraoperative dual cortical stimulation. Brain Stimul. (2020) 13:819-31. doi: 10.1016/j.brs.2020.02.023n

16. Giampiccolo D, Basaldella F, Badari A, Squintani M, Cattaneo L, Sala F. Feasibility of cerebello-cortical stimulation for intraoperative neurophysiological monitoring of cerebellar mutism. Child's Nerv Syst. (2021) 37:1505-14. doi: 10.1007/s00381-021-05126-7n

17. Yamao Y, Matsumoto R, Kunieda T, Arakawa Y, Kobayashi K, Usami $\mathrm{K}$, et al. Intraoperative dorsal language network mapping by using single-pulse electrical stimulation. Hum Brain Mapp. (2014) 35:434561. doi: 10.1002/hbm.22479n

18. Giampiccolo D, Parmigiani S, Basaldella F, Russo S, Pigorini A, Rosanova $\mathrm{M}$, et al. Recording cortico-cortical evoked potentials of the human arcuate fasciculus under general anaesthesia. Clin Neurophysiol. (2021) 132:13323 doi: 10.1016/j.clinph.2021.03.044n

19. Fernández-Miranda JC, Wang Y, Pathak S, Stefaneau L, Verstynen T, Yeh FC. Asymmetry, connectivity, and segmentation of the arcuate fascicle in the human brain. Brain Struct Funct. (2015) 220:166580. doi: 10.1007/s00429-014-0751-7n

20. Koch G, Fernandez Del Olmo M, Cheeran B, Ruge D, Schippling S, Caltagirone $\mathrm{C}$, et al. Focal stimulation of the posterior parietal cortex increases the excitability of the ipsilateral motor cortex. J Neurosci. (2007) 27:681522. doi: 10.1523/JNEUROSCI.0598-07.2007n

21. Koch G. Cortico-cortical connectivity: the road from basic neurophysiological interactions to therapeutic applications. Exp Brain Res. (2020) 238:167784. doi: 10.1007/s00221-020-05844-5n

22. Patton HD, Amassian VE. Single- and multiple-unit analysis of cortical stage of pyramidal tract activation. I Neurophysiol. (1954) 17:34563. doi: 10.1152/jn.1954.17.4.345n

23. Yamamoto T, Katayama Y, Nagaoka T, Kobayashi K, Fukaya C. Intraoperative monitoring of the corticospinal motor evoked potential (D-wave): clinical index for postoperative motor function and functional recovery. Neurol Med Chir. (2004) 44:170-82. doi: 10.2176/nmc.44.170n

24. Fujiki M, Furukawa Y, Kamida T, Anan M, Inoue R, Abe T, et al. Intraoperative corticomuscular motor evoked potentials for evaluation of motor function: a comparison with corticospinal D and I waves. J Neurosurg. (2006) 104:8592. doi: $10.3171 /$ jns.2006.104.1.85n

25. Deletis V, Sala F. Intraoperative neurophysiological monitoring of the spinal cord during spinal cord and spine surgery: a review focus on the corticospinal tracts. Clin Neurophysiol. (2008) 119:248-64. doi: 10.1016/j.clinph.2007.09.135n

26. Hallett M, Di Iorio R, Rossini PM, Park JE, Chen R, Celnik P, et al. Contribution of transcranial magnetic stimulation to assessment of brain connectivity and networks. Clin Neurophysiol. (2017) 128:212539. doi: 10.1016/j.clinph.2017.08.007n

27. Deletis V, Rodi Z, Amassian VE. Neurophysiological mechanisms underlying motor evoked potentials in anesthetized humans. Part 2. relationship between epidurally and muscle recorded MEPs in man. Clin Neurophysiol. (2001) 112:445-52. doi: 10.1016/S1388-2457(00)00557-5n

28. Ugawa Y, Uesaka Y, Terao Y, Hanajima R, Kanazawa I. Magnetic stimulation over the cerebellum in humans. Ann Neurol. (1995) 37:70313. doi: 10.1002/ana.410370603n

29. Daskalakis ZJ, Paradiso GO, Christensen BK, Fitzgerald PB, Gunraj C, Chen R. Exploring the connectivity between the cerebellum and motor cortex in humans. J Physiol. (2004) 557:689-700. doi: 10.1113/jphysiol.2003.059808n
30. Grodd W, Hülsmann E, Lotze M, Wildgruber D, Erb M. Sensorimotor mapping of the human cerebellum: fMRI evidence of somatotopic organization. Hum Brain Mapp. (2001) 13:55-73. doi: 10.1002/hbm.1025n

31. Manni E, Petrosini L. A century of cerebellar somatotopy: a debated representation. Nat Rev Neurosci. (2004) 5:241-9. doi: 10.1038/nrn1347n

32. Adrian ED. The spread of activity in the cerebral cortex. J Physiol. (1936) 88:127-61. doi: 10.1113/jphysiol.1936.sp003427n

33. Matsumoto R, Nair DR, LaPresto E, Najm I, Bingaman W, Shibasaki H, et al. Functional connectivity in the human language system: a cortico-cortical evoked potential study. Brain. (2004) 127:2316-30. doi: 10.1093/brain/awh246n

34. Keller CJ, Bickel S, Entz L, Ulbert I, Milham MP, Kelly C, et al. Intrinsic functional architecture predicts electrically evoked responses in the human brain. Proc Natl Acad Sci USA. (2011) 108:1030813. doi: 10.1073/pnas.1019750108n

35. Valentin A, Anderson M, Alarcon G, Seoane JG, Selway R, Binnie CD, et al. Responses to single pulse electrical stimulation identify epileptogenesis in the human brain in vivo. Brain. (2002) 125:1709-18. doi: 10.1093/brain/awf187n

36. Silverstein BH, Asano E, Sagiura A, Sonoda M, Lee, M.-H, Jeong J.-W. Dynamic tractography: integrating cortico-cortical evoked potentials and diffusion imaging. Neuroimage. (2020) 215:116763. doi: 10.1016/j.neuroimage.2020.116763n

37. Entz L, Tóth E, Keller CJ, Bickel S, Groppe DM, Fab,ó D, et al. Evoked effective connectivity of the human neocortex. Hum Brain Mapp. (2014) 35:5736-53. doi: 10.1002/hbm.22581n

38. Shine JM, Kucyi A, Foster BL, Bickel S, Wang D, Liu H, et al. Distinct patterns of temporal and directional connectivity among intrinsic networks in the human brain. J Neurosci. (2017) 37:9667-74. doi: 10.1523/JNEUROSCI.1574-17.2017n

39. Matsumoto R, Nair DR, LaPresto E, Bingaman W, Shibasaki H, Lüders HO. Functional connectivity in human cortical motor system: a cortico-cortical evoked potential study. Brain. (2007) 130:181-97. doi: 10.1093/brain/awl257n

40. Enatsu R, Gonzalez-Martinez J, Bulacio J, Kubota Y, Mosher J, Burgess RC, et al. Connections of the limbic network: a corticocortical evoked potentials study. Cortex. (2015) 62:20-33. doi: 10.1016/j.cortex.2014.06.018n

41. Suzuki Y, Enatsu R, Kanno A, Yokoyama R, Suzuki H, Tachibana S, et al. The Influence of anesthesia on corticocortical evoked potential monitoring network between frontal and temporoparietal cortices. World Neurosurg. (2019) 123:e685-92. doi: 10.1016/j.wneu.2018.11.253n

42. Saito T, Tamura M, Muragaki Y, Maruyama T, Kubota Y, Fukuchi S, et al. Intraoperative cortico-cortical evoked potentials for the evaluation of language function during brain tumor resection: initial experience with 13 cases. J. Neurosurg. (2014) 121:827-38. doi: 10.3171/2014.4.JNS131195n

43. Martino J, De Witt Hamer PC, Berger MS, Lawton MT, Arnold CM, De Lucas EM, et al. Analysis of the subcomponents and cortical terminations of the perisylvian superior longitudinal fasciculus: a fiber dissection and DTI tractography study. Brain Struct Funct. (2013) 218:10521. doi: 10.1007/s00429-012-0386-5n

44. Yagmurlu K, Middlebrooks EH, Tanriover N, Rhoton AL. Fiber tracts of the dorsal language stream in the human brain. J Neurosurg. (2016) 124:1396405. doi: 10.3171/2015.5.JNS15455n

45. Nakae T, Matsumoto R, Kunieda T, Arakawa Y, Kobayashi K, Shimotake A, et al. Connectivity gradient in the human left inferior frontal gyrus: intraoperative cortico-cortical evoked potential study. Cereb Cortex. (2020) 30:4633-50. doi: 10.1093/cercor/bhaa065n

Conflict of Interest: The authors declare that the research was conducted in the absence of any commercial or financial relationships that could be construed as a potential conflict of interest.

Copyright (c) 2021 Sala, Giampiccolo and Cattaneo. This is an open-access article distributed under the terms of the Creative Commons Attribution License (CC BY). The use, distribution or reproduction in other forums is permitted, provided the original author(s) and the copyright owner(s) are credited and that the original publication in this journal is cited, in accordance with accepted academic practice. No use, distribution or reproduction is permitted which does not comply with these terms. 